\title{
Contabilidad del Conocimiento: UNA NECESIDAD IMPERIOSA EN LA Contabilidad en el Perú
}

\author{
Dr. Ernesto Polar Falcón \\ Docente principal. \\ CPC.Miguel Nicolás Diaz Inchicaqui \\ Docente asociado
}

\begin{abstract}
RESUMEN
La investigación titulada: "La Contabilidad del Conocimiento: una necesidad imperiosa en la contabilidad en el Perú" analiza la problemática actual de la ciencia contable como parte del sistema de información empresarial que manejan los gestores de negocios en la nueva economía del conocimiento, considerando que en estas últimas décadas se ha producido un cambio muy importante con relación a los factores generadores de beneficios en las empresas. Es decir, de una economía basada en la producción que consideraba a los activos físicos y financieros como su fuente principal generadora de recursos, se ha pasado a una economía basada en el conocimiento. En el marco de esta nueva visión de la economía ha cobrado importancia una nueva concepción denominada: el capital intelectual, que representa un desafío para la contabilidad tradicional al no contabilizarse actualmente este activo intangible. Este reto preocupante para la profesión contable debe ser superado, a nuestro entender, con la revisión de las prácticas de exposición de la información financiera, para concebir su utilidad por los usuarios en la toma de decisiones empresariales. Este desafío profesional es tomado en cuenta en esta investigación ya que en nuestro país aún no se ha divulgado de una manera masiva los aspectos conceptuales de la contabilidad del conocimiento, habiéndose considerado sólo las convenciones contables adaptadas y aprobadas en la NIC 38 Intangibles emitidas por la Contaduria Pública de la Nación. Al final de este trabajo nuestra propuesta de un nuevo modelo de visión de la contabilidad en la cual identificamos no sólo el capital intelectual sino también proponemos un modelo de ajuste contable para ser registrado y expuesto en notas complementarias a los estados financieros que se emitan.
\end{abstract}

Palabras clave: Valor agregado, ventaja competitiva, capital intelectual, procesos organizacionales, activos intangibles, posición dominante, modelo proactivo.

\section{MARCO TEÓRICO DE LA CONTABILIDAD DEL CONO- CIMIENTO}

\subsection{LOS NEGOCIOS EN LA NUEVA ECONOMÍA}

Alvin Toffler en su obra La Tercera Ola, manifiesta que la sociedad ha pasado a una etapa de transición y revolución en la economía mundial, la misma que está dejando atrás la generación de riquezas a través de bienes de capital y productos manufacturados, para reemplazarlos por bienes menos tangibles como el conocimiento y los procesos de información. Si analizamos esta afirmación podemos admitir que los viejos pilares de la economía: tierra, capital y trabajo quedaron desvirtuados ante el advenimiento de nuevas empresas que basan su producción en el conocimiento y la tecnología.

Entonces hoy estamos ante empresas o negocios con características de multiplicidad, variabilidad, velocidad y complejidad, ya que no dependen solo de un deudor $y$ un acree- 
dor. En términos económicos, los negocios de estar centrados sólo en el intercambio comercial y productivo, han pasado a ser empresas que giran alrededor de la generación y agregación de valor a través de los servicios que brindan, la información y conocimiento que generan como ventaja competitiva.

A esta variable moderna que genera valor a las empresas se le ha llamado Capital Intelectual.

En el marco de esta nueva visión de la economía, el Capital Intelectual se presenta como un desafío para la contabilidad tradicional, que está bajo los conceptos de partida doble, normatividad legal y convenciones contables vigentes y que han dejado de ser importantes para la toma de decisiones empresariales. En conclusión, el mundo de hoy no gira sólo alrededor del derecho y la economía, gira además alrededor de la administración que cumple con sus objetivos y maneja sus recursos de manera eficiente, basadas en la información oportuna a través de sistemas de tecnologías de la información que pueden ser generadas interna y externamente, con una radical orientación hacia el mayor uso del conocimiento.

\subsection{PROBLEMÁTICA MUNDIAL DE LA CONTABILIDAD TRADICIONAL}

La contabilidad tradicional centra sus criterios de valoración y medición en principios contables generalmente aceptados, tales como: el costo histórico, el valor de mercado, el valor de reposición y el valor de realización; pero ¿puede la contabilidad tradicional medir la imaginación y creatividad de los ingenieros de la empresa responsables de la creación de nuevos productos?, o ¿¿de los procesos de información que dotan a los empleados de las herramientas necesarias para decidir hacer una venta, realizar un pedido, ingresar un producto en un mercado? o más aún ¿pueden estos principios medir el conocimiento adquirido por los empleados y obreros de la empresa en la realización de sus labores cotidianas, aspecto que les da cierto grado de especialización y dominio a la empresa sobre otras?; como ejemplo, podemos citar en el Perú a Telefónica del Perú, que basa los servicios que brinda en soportes tecnológicos y conocimientos de telecomunicaciones para cumplir con sus objetivos organizacionales, estos últimos no son tomados en cuenta por la contabilidad financiera tradicional.

Si vemos con realismo que los negocios han sufrido grandes cambios en el manejo de la información para la toma de decisiones, podemos inferir que la contabilidad tradicional debe ser modificada sustancialmente en sus técnicas y aplicaciones científicas para estar a la par de ella. Es por ello, que la profesión contable viene enfrentando un reto importante vinculado con la revisión de las prácticas de exposición de la información financiera útil a los inversionistas, ya que hace cierto tiempo que las organizaciones empresariales se dieron cuenta que sus activos físicos y financieros no tienen la capacidad de generar ventajas competitivas sostenibles en el tiempo, descubriéndose entonces que los activos intangibles (Capital Intelectual) son los que aportan verdadero valor a las operaciones que realizan.

En conclusión, los estados financieros básicos que emite la Contabilidad se pueden resumir en dos, por su mayor importancia: El Balance General que muestra la posición financiera de la empresa a una fecha determinada y el Estado de Ganancias y Pérdidas que contiene el resultado económico de ese mismo período, no pueden dar respuesta a las siguientes preguntas: ¿los estados financieros 
tradicionales pueden mostrar el desempeño real que ha tenido la empresa en el uso de sus inversiones para generar valor en sus resultados?, ¿pueden medir el capital intelectual que posee la empresa y sobre el cual hace uso para gestionar sus actividades y conseguir los resultados económicos expresados en el Estado de Ganancias y Pérdidas?

\subsection{LA CONTABILIDAD DEL CONOCIMIENTO}

La contabilidad del conocimiento surge como respuesta a los problemas derivados de la contabilidad tradicional al no poder generar información para administrar el capital intelectual (gestión del conocimiento).

Para llegar a conceptuar qué es la contabilidad del conocimiento, en primer lugar determinemos ¿qué es el conocimiento?, pregunta que ha sido analizada por las mentes más privilegiadas del pensamiento occidental, desde Aristóteles y Platón hasta la actualidad. Recordemos una cita de Platón: "Supongamos ahora que en la mente de cada hombre hay una pajarera con toda suerte de pájaros. Algunos en bandadas aparte de los demás, otros en pequeños grupos, otros a solas, volando de aquí para allá por todas partes... podemos suponer que los pájaros son tipos de conocimien to y que cuando éramos niños este receptáculo estaba vacío; cada vez que un hombre obtiene y encierra en la jaula una clase de conocimiento, se puede decir que ha aprendido o descubierto la cosa que es el tema del conocimiento; y en esto consiste el saber".

El diccionario define conocimiento como: "El producto o resultado de ser instruido, el conjunto de cosas sobre las que se sabe o que están contenidas en la ciencia". El diccionario acepta que la existencia de conocimiento es muy difícil de observar y reduce su presencia a la detección de sus efectos posteriores, los conocimientos se almacenan en la persona (o en otro tipo de agentes). Esto hace que sea casi imposible observarlos. Como vemos existen múltiples definiciones de conocimiento, desde las clásicas y fundamentales como una creencia cierta y justificada, a otras más recientes y pragmáticas como una mezcla de experiencia, valores, información y saber hacer, que sirve como marco para la incorporación de nuevas experiencias e información, y es útil para la acción. De todo ello, podríamos resumir que las características del conocimiento son:

- El conocimiento es personal, en el sentido de que se origina y reside en las personas, que lo asimilan como resultado de su propia experiencia (es decir, de su propio hacer, ya sea físico o intelectual) y lo incorporan a su acervo personal estando convencidas de su significado e implicaciones, articulándolo como un todo organizado que da estructura y significado a sus distintas piezas.

- Su utilización, que puede repetirse sin que el conocimiento se consuma como ocurre con otros bienes físicos, permite entender los fenómenos que las personas perciben (cada una a su manera, de acuerdo precisamente con lo que su conocimiento implica en un momento determinado), y también evaluarlos, en el sentido de juzgar la bondad o conveniencia de los mismos para cada una en cada momento; $y$

- sirve de guía para la acción de las personas, en el sentido de decidir qué hacer en cada momento porque esa acción tiene en general por objetivo mejorar las consecuencias para cada individuo de los fenómenos percibidos (incluso cambiándolos si es posible).

Estas características convierten al conocimiento, cuando en él se basa la oferta de una 
empresa en el mercado, en un cimiento sólido para el desarrollo de sus ventajas competitivas. En efecto, en la medida en que es el resultado de la acumulación de experiencias de personas, su imitación es complicada a menos que existan representaciones precisas que permitan su transmisión a otras personas efectiva y eficientemente.

En conclusión, para dar lugar a la contabilidad del conocimiento primeramente debemos concebir al conocimiento como un recurso o activo de la empresa que constituye un alto valor y fuente de riqueza que debe administrarse mediante procesos organizacionales, por su reconocimiento como factor crucial de mejora de la producción y servicios al cliente, que mejoran el desempeño de la organización, dinamizándola y generando o agregando valor en las operaciones que ésta realiza. En segundo lugar, reconocer que éstos son intangibles que tienen características específicas conocidas como Capital Intelectual, las mismas que son reconocidas en el IAS $38 \mathrm{y}$ específicamente en el Estudio 7 del IFAC titulado La medición y gerenciamiento del Capital Intelectual: una introducción, que reconoce en los modelos desarrollados por la doctrina internacional que el capital intelectual se puede conceptuar como la suma de tres componentes que se interrelacionan para la formación del valor:

a) Capital Humano: formado por las habilidades, educación, calificación personal, conocimientos relativos al trabajo, tasa ocupacional, tasa psicometría, capacidades relacionadas con el trabajo, empuje empresarial, habilidades de acción y reacción para las innovaciones, flexibilidad, etc.

b) Capital clientela: formado por las marcas de fabrica, clientes, lealtad de los clientes, nombres de la empresa, acumulación de pedidos, canales de distribución, colaboraciones de negocios, acuerdos de licencias, contratos favorables, acuerdos de franchising, etc.

c) Capital Organizacional (estructural): dividido a su vez en:

- Propiedad intelectual: conformada por las patentes, los derechos de edición, los derechos de diseño, las fórmulas secretas, las marcas comerciales y las marcas de servicios.

- Infraestructura de activos: donde se considera la filosofía de gerenciamiento, la cultura corporativa, el proceso de gerenciamiento, los sistemas de información, los sistemas de redes de trabajo y las relaciones financieras.

Hasta ahora se ha intentado establecer qué elementos integran esta expresión que parece haberse puesto de moda en los últimos tiempos: Capital Intelectual. Sin duda, se trata de intangibles imprescindibles para la creación de valor en las empresas y que le otorgan a las mismas ventajas comparativas en relación a otras. En base a lo expuesto se establecen los siguientes componentes del capital intelectual:

a) Acrivos de mercado: comprenden las marcas, denominación social de la empresa, fidelidad de la clientela, reserva de pedidos, canales de distribución, licencias y franquicias. En definitiva, son elementos que proporcionan a la empresa una ventaja competitiva en el mercado.

b) Activos de propiedad intelectual: incluyen elementos como conocer a la empresa, secretos de fabricación, derechos de autor, patentes, derechos de diseño y marcas de fábricas y servicios.

c) Activos de infraestructuras: comprenden elementos que definen la forma de trabajo en la organización, filosofía de la gestión, cultura corporativa, procesos de gestión, tecnología de la información, sistemas de interconexión y relaciones financieras. 
d) Activos centrados en el individuo: incluyen elementos tales como el nivel de estudios alcanzado, calificaciones profesionales, conocimientos técnicos, psicometría asociado con el trabajo.

Estos elementos, a diferencia de los activos de mercado, propiedad intelectual e infraestructura, no pueden ser de propiedad de la empresa.

Por lo tanto, son activos intangibles todo aquello que la organización utiliza para generar valores tales como: las capacidades que se generan en la organización cuando los recursos empiezan a trabajar en grupo y coordinadamente. Para ello, la contabilidad del conocimiento debe explicar y sistematizar los procesos asociados a su creación, recolección, organización, difusión, uso y explotación, transformando el conocimiento personal en corporativo que debe ser distribuido ampliamente en toda la organización y aplicado de manera apropiada. Entonces, hoy el problema radica en disponer de medidas comprensibles que sean útiles para su reconocimiento, valorización y revelación. En el siguiente cuadro resumimos este tipo de activos: miento como aquellos inherentes a la relación de la organización con su entorno y a la utilización eficiente de su estructura operativa. Es en esta necesidad que se plantean los modelos de medición y control del capital intelectual que buscan resolver el valor real de los activos que no se registran contablemente y que usualmente son llamados fondo de comercio, valor agregado de mercado, conocimiento de la empresa, capital intelectual, ya que el valor real de una empresa no coincide con lo que dicen sus balances y registros contables.

Desde la década del setenta, y con mucha más fuerza a partir del último quinquenio del siglo pasado, han surgido sistemas de control que, en su proceso integracional, no se contentan con desarrollar indicadores financieros, sino que avanzan sobre elementos de la empresa que no son tomados en cuenta por la contabilidad tradicional. Estos sistemas, conocidos entre otros son:

a) El Balanced Scorecard. Creado por Robert Kaplan y David Norton, manifiesta que los activos no reconocidos contablemente, entre los que se encuentran los referentes

\begin{tabular}{|c|c|c|c|c|}
\hline \multirow{3}{*}{$\begin{array}{c}\text { ACTIVOS } \\
\text { INTANGIBLES } \\
\text { VISIBLES }\end{array}$} & \multicolumn{2}{|c|}{ TIPOS } & \multirow{3}{*}{$\begin{array}{c}\text { DISPOSICIONES } \\
\text { IASC } \\
\text { FASB } \\
\text { PCGA } \\
\text { AECA } \\
\text { ICAC }\end{array}$} & $\begin{array}{l}\text { TRATAMIENTO } \\
\text { CONTABLE }\end{array}$ \\
\hline & $\begin{array}{c}\text { Identificables y } \\
\text { controlables }\end{array}$ & $\begin{array}{c}\text { Marcas, gastos } \\
\text { de I+D }\end{array}$ & & \multirow{2}{*}{$\begin{array}{l}\text { Analizar partidas } \\
\text { por partidas } \\
\text { discrepancias y } \\
\text { normativas }\end{array}$} \\
\hline & $\begin{array}{c}\text { No } \\
\text { identificables y } \\
\text { controlables }\end{array}$ & $\begin{array}{l}\text { Fondo de } \\
\text { Comercio }\end{array}$ & & \\
\hline $\begin{array}{l}\text { ACTIVOS } \\
\text { INTANGIBLES } \\
\text { OCULTOS }\end{array}$ & $\begin{array}{l}\text { Capital } \\
\text { intelectual }\end{array}$ & $\begin{array}{c}\text { Humano } \\
+ \\
\text { Estructural } \\
+ \\
\text { Clientela } \\
\end{array}$ & \multicolumn{2}{|c|}{ Sin regulación } \\
\hline
\end{tabular}

\subsection{MODELOS DE MEDICIÓN Y ADMINISTRACIÓN DEL CAPITAL INTELECTUAL}

Los responsables de los negocios y aquellos que buscan invertir en ellos, necesitan información relacionada con el valor de los activos intangibles, referido tanto al conoci- al capital intelectual deben ser medidos a través de indicadores, ya que lo que no se mide no se gestiona y siendo los activos intangibles un factor importante de la gestión empresarial, es comprensible su estudio y evaluación bajo cuatro perspectivas planteadas: 
- Perspectiva financiera: los indicadores financieros tales como: rentabilidad sobre la inversión, flujos de caja, análisis de la rentabilidad de clientes y productos, gestión de riesgo, entre otros deben ser complementados con otros asociados a la realidad empresarial.

- Perspectiva del cliente: Debe identificar los valores relacionados con los clientes que incrementan la capacidad competitiva de la empresa. Para ello debemos realizar un análisis del valor y calidad de éstos en el segmento de mercado objetivo.

- Perspectiva de los procesos internos: analiza cómo se adecúan los procesos de la empresa para lograr la satisfacción del cliente y conseguir con ello los niveles de rendimiento financiero planificados. En estos procesos internos distinguimos tres tipos de procesos: de innovación, operaciones y servicios postventa.

- Perspectiva del aprendizaje y crecimiento: presenta al conjunto de indicadores que constituyen los activos que dotan a la organización de la habilidad para mejorar y aprender, tales como: capacidad y competencia de personas, sistemas de información, cultura-clima-motivación para el aprendizaje y la acción. b) Modelo de valoración de negocios. Parte de la misma base e indica que se debe ir más allá de los números tradicionales y ayudar a implementar la estrategia. Está estructurado en tres niveles:

- Cualidad de liderazgo

- = Administración

- Creación de valor

- = Operaciones + desempeño

- Generación de ingresos

- = Negocio central + Mercado + Competencia

c) Valor Económico Agregado (EVA) y Valor de Mercado'Agregado (MVA). Es una medida de desempeño financiero que combina el concepto de ingreso residual con los principios de las finanzas corporativas modernas. Específicamente, EVA es la utilidad operacional después de impuestos menos un gasto de capital. De acuerdo con este índice, si la rentabilidad/retorno sobre el capital de la empresa sobrepasa sus costos de capital se está creando verdadero valor para los accionistas. Este índice mezcla tres elementos importantes:

- El flujo de efectivo

- El efectivo medido durante un período (duración del crecimiento del valor)

- Riesgo conocido como costo de capital.

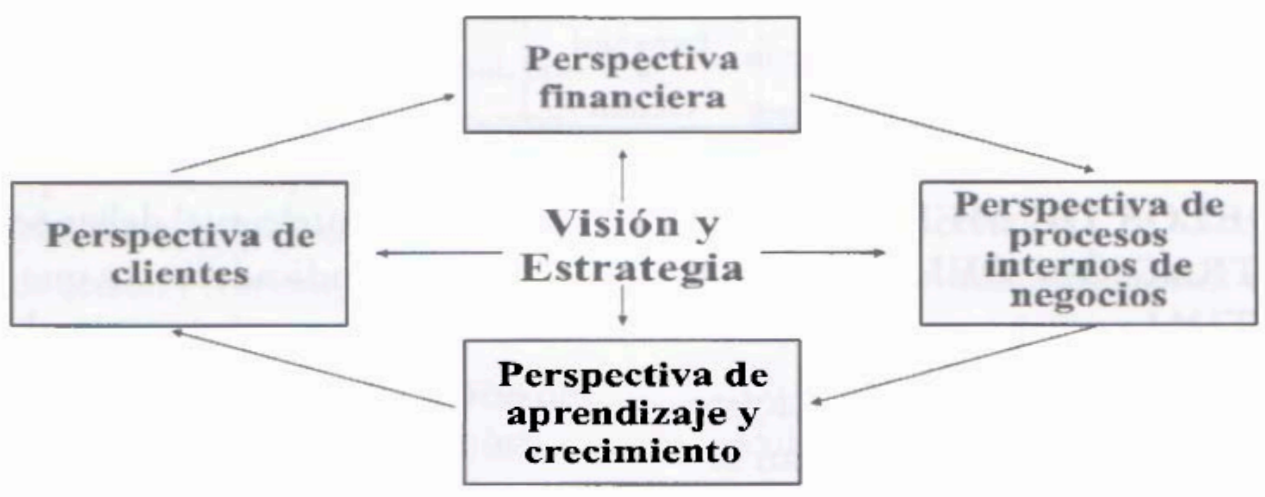

Figura: Balanced Scorecad

Fuente: Kaplan y Norton (1996) 
II. LA CONTABILIDAD DEL CONOCIMIENTO APLICACIONES TÉCNICAS Y CIENTÍFICAS A LA REALIDAD PERUANA

Nuestra investigación tiene como objetivo fundamental reconocer las limitaciones de la contabilidad tradicional vigente en el Perú, y, a partir de ello, plantear soluciones técnicas y científicas tal como se expresan a continuación:

\subsection{LIMITACIONES DE LA CONTABILIDAD VIGENTE EN EL PERÚ}

En el Perú, tradicionalmente, la contabilidad financiera ha tenido su arraigo en enfoques eminentemente jurídicos y financieros, es decir, aplicamos vehementemente el concepto jurídico de propiedad, de allí la palabra patrimonio que está insertada en los estados financieros tradicionales. Otro aspecto es que, hasta hoy, la contabilidad viene utilizando métodos de valoración desarrollados en la economía para establecer los precios de transacciones. Hecho comprensible en el marco de una interpretación contable de naturaleza económica.

Si vamos a su concepción más profunda, en la contabilidad se han utilizado fundamentalmente la teoría del valor utilidad y del valor del trabajo.

- La teoría del valor utilidad: establece que el valor de un bien se determina por la capacidad de satisfacción de necesidades, hecho que implica que es la demanda de bienes en el mercado el determinante del valor. Sólo tienen valor los bienes destinados al mercado, siendo este último el que determine el precio de un bien o servicio. Esta teoría se expresa en contabilidad a través de los valores de mercado, compra, reposición o realización.
- La teoría del valor del trabajo: según la cual el valor de un bien se determina por el trabajo socialmente necesario para ponerlo en disposición de consumo, como las remuneraciones acumuladas en los procesos de explotación, producción y distribución; de allí nace el concepto de costo histórico constituido por las remuneraciones al trabajo y al capital, y si incorporamos el concepto marxista según el cual el trabajo es el único creador de valor, se niega la existencia de otro tipo de valoraciones.

En ese sentido la contabilidad financiera que desarrollamos se convierte en un serio obstáculo al abordar el concepto de valoración, por su óptica eminentemente restringida, ya que si preguntáramos a contadores tradicionales, ¿cuáles son los activos de una empresa? éste miraría el balance de la empresa y nos indicaría el valor de los edificios, instalaciones, capital social... en suma, los activos materiales tangibles, elementos todos ellos basados en un solo factor: el financiero o económico. Sin embargo, estos elementos no suponen una ventaja competitiva para las empresas, ya que los tiempos en los que la posición dominante de una empresa en el mercado se basaba en su tamaño de inversión han pasado. ¿Dónde reside entonces esta ventaja?, ¿en la tecnología? Si bien la innovación tecnológica es fundamental para sobrevivir, no es menos cierto que dicha tecnología cada vez más se encuentra al alcance de todos y es fácilmente copiable. De hecho, países enteros como Corea o Singapur han basado su estrategia de crecimiento nacional en el traslado e imitación de la tecnología desarrollada en otros países (principalmente Japón) ... y no les van tan mal. No estamos, por lo tanto, ante una ventaja competitiva sino más bien frente a una desventaja: quien no disponga de esa tecnología perecerá, pero 
quien disponga de ella no gozará de una posición ventajosa respecto a los demás.

Si ni los activos materiales ni la tecnología son, pues, la base de la ventaja competitiva de una organización, ésta debe hallarse en otros activos que maneja la empresa: ¿cuáles? Es ante esta pregunta que pensamos que el valor de una organización ya no reside en sus bienes tangibles, sino en los conocimientos técnicos y especializados de su personal, en su experiencia, en la propiedad intelectual, la fidelidad de los clientes,...en resumen, en lo que se ha venido en llamar capital intelectual o conocimiento.

Pongamos un pequeño ejemplo. Backus y el monopolio que tiene a nivel nacional con la corporación de empresas cerveceras, ahora ve amenazada su participación en el mercado con el probable ingreso de la poderosa cervecera Ambev de capitales brasileños. Es decir estamos ante dos empresas con inversión en activos fijos con tecnología de última generación y solidez financiera. Sin embargo, de acuerdo con el análisis realizado, cuando encren en competencia estas dos empresas, Backus tendrá a su favor la experiencia (tanto de su personal como corporativa) en materias como el conocimiento del sector en el país y la utilización de estrategias en el tratamiento de canales de distribución, lealtad de clientes que no podría, en ningún caso, ser imitado o copiado por la competencia, salvo que se produjera una "fuga" de personal (lo cual nos lleva a otro campo interesantísimo y con creciente importancia, el cual es la fidelización de dicho personal). Entonces, su memoria corporativa sería y es su ventaja competitiva.

En nuestro país la Contaduría Pública de la Nación no ha llevado a cabo investigaciones a través del Consejo Normativo de Contabilidad, el cual ha aprobado las Normas
Internacionales de Contabilidad, como es el caso de la NIC 38 en base al IAS 38 y el estudio 7 del IFAC, que define a los activos intangibles susceptibles a ser valorados e incluidos en la Contabilidad, confirmándose de esta manera la insuficiencia del modelo contable actual para valorar y exponer el capital intelectual que la empresa maneja y el cual contribuye al logro y sostenimiento de sus ventajas competitivas. Según dicha norma, un activo es un recurso:

- Controlado por una empresa como resultado de eventos pasados por el cual se espera que fluyan beneficios económicos futuros para una empresa.

Una limitante importante de IAS 38 es que debajo del modelo tradicional de contabilidad, se reconoce un activo intangible si:

- Los beneficios económicos futuros fueran atribuibles al activo que fluirá para la empresa.

- Se puede medir confiablemente el costo del activo.

Bajo este criterio, se continúa con las restricciones del modelo tradicional de contabilidad, es decir, no sería visible medir aspectos como: marcas, fidelidad de los clientes y relaciones establecidas, good will (cuando no ha sido adquirido directamente por la empresa), conocimiento acumulado en las personas, etc. Es decir es un modelo limitado, que se fundamenta en los modelos de valorización de costos históricos.

\subsection{SOLUCIONES TÉCNICAS Y CIENTÍFICAS A LA REALIDAD PERUANA}

a) Identificación del capital intelectual y presentación de un nuevo modelo para su aplicación en la contabilidad del conocimiento. 
Siendo la contabilidad el lenguaje de los negocios y dada la evolución de éstos en las últimas décadas, nos queda muy claro que la contabilidad debe de cambiar, ya que algunos elementos definitivamente pertenecen al pasado y hoy ya no sirven, dadas las circunstancias actuales de los negocios y de las organizaciones. Es obvio que los negocios cambiaron, actualmente son múltiples y variados, veloces, casi frenéticos y complejos; es decir, ya no son solamente un deudor y un acreedor, tal como lo indicamos en párrafos anteriores. En términos económicos ya no está centrado sólo en el intercambio comercial o en la producción, hoy giran en la generación del valor agregado en sus servicios, información y conocimiento.

Es decir del modelo tradicional estático de la contabilidad tradicional:
Debemos de pasar a un modelo proactivo y futurista de la contabilidad que nos guíe no sólo en nuestra manera de pensar, sino en la ubicación, localización, funciones y resultados del capital intelectual que manejen las empresas para su posterior presentación en los estados financieros que incluya ésta. Para ello debemos de presentar el modelo sugerido de la nueva visión de la contabilidad:

- La potencialidad. La potencialidad de los negocios depende de su estructura organizacional o capacidad operativa, que está compuesta por los activos que mantiene la empresa como inversión (vida útil para proyectar el negocio al futuro) y los pasivos o capitales financieros que financia la misma. Esta variable estática es producto de la contabilidad tradicional, que al asociarse con los clientes o mercado

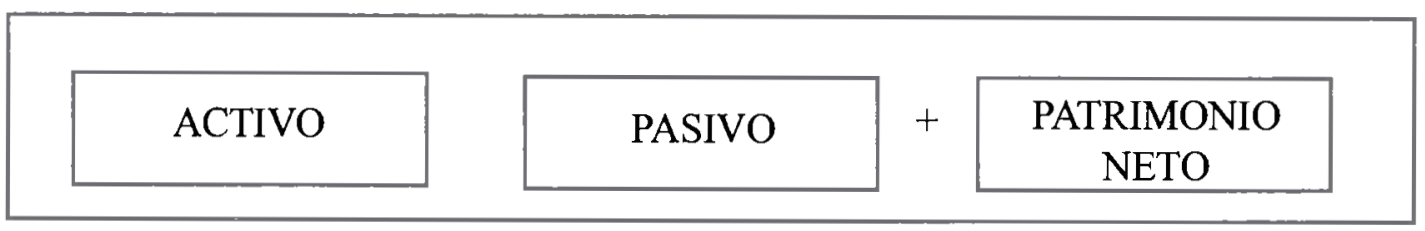

Al replantear la fórmula contable tradicional, fundamentamos nuestra teoría en la "perspectiva contable de justificar la diferencia entre el valor de mercado y el valor contable", en estas tres variables. Para ello, definiremos, analizaremos el contenido y el significado de cada una de éstas con la finalidad de llegar a un entendimiento del proceso de valorización de la empresa que incluya el capital intelectual. puede medir sus resultados financieros a través del estado de ganancias y pérdidas.

- La performance. Significa lo que se debe hacer, esta variable la fundamentamos en la Teoría de los recursos y capacidades que tiene la empresa para hacer una gestión o desempeño óptimo. En efecto, ante entornos turbulentos, con incertidumbre, complejidad, competencia global, acortamiento del ciclo de vida de los produc-

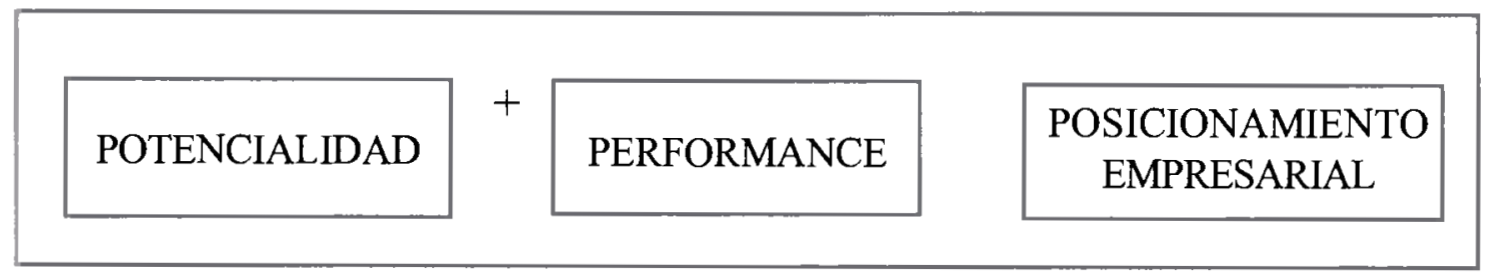


tos, cambios rápidos en los gustos y las necesidades de los consumidores, etc., la empresa tiene dificultades para plantearse qué necesidades quiere satisfacer, por lo que, puede preguntarse alternativamente, acerca de qué necesidades puede satisfacer. En este último caso, la orientación externa no puede ser el único fundamento de la estrategia empresarial, sino que es preciso recurrir al análisis de los recursos y capacidades disponibles para poner en marcha una estrategia.

Bajo el criterio definido en el párrafo anterior, para que funcione esta variable se debe partir seleccionando una estrategia que explote los recursos y capacidades de la empresa, asegurando el uso total de ellos para llegar al beneficio óptimo planificado. Es decir, esta variable dinamiza la potencialidad del negocio y determina como resultado el valor agregado de la organización hacia sus clientes. Por consiguiente, si hablamos de la organización y su actuación en los diferentes niveles ya sea definiendo estrategias y cumpliendo los objetivos corporativos estimados, consideramos que es en esta variable que se ubica el capital intelectual.

La performance debe ser evaluada en:

a) El capital humano (recursos humanos), el capital estructural (procesos, tecnología) y el capital relacional (los clientes).

b) La administración de la información dentro de la organización.

c) La participación del conocimiento como actividad y útil para las necesidades estratégicas de la empresa.

d) El aprendizaje continuo.

Estos elementos de la performance sólo tienen valor al combinarse con la inversión de la empresa u otros activos netos que denominamos potencialidad. Presentamos seguidamente una matriz que nos ayudará a conocer las causas que son determinantes en el desempeño de la empresa, la misma que según nuestro modelo sugerido al ser evaluado determinará una performance positiva o negativa.

Cuando la performance es positiva, nuestra propuesta es reconocer los gastos contabilizados como parte de la inversión al haberse reconocido y validado en el desempeño de la empresa los activos intangibles provenientes de competencias personales, estructura interna y estructura externa.

- Posicionamiento empresarial. O valor de la empresa que debe ser cuantificado continuamente ya que ésta es el resultado de las ventajas competitivas generadas por el capital intelectual que maneja la empresa.

\begin{tabular}{|c|c|c|}
\hline EFECTO & CAUSA & CAUSA \\
\hline \multirow{4}{*}{$\begin{array}{l}\text { DESEMPEÑO DE LA } \\
\text { EMPRESA }\end{array}$} & $\begin{array}{l}\text { FACILITADORES que proveen dirección y } \\
\text { naturaleza para las acciones. }\end{array}$ & $\begin{array}{l}\text { Conocimiento } \\
\text { Activos intelectuales }\end{array}$ \\
\hline & $\begin{array}{l}\text { FACILITADORES que proveen soporte para las } \\
\text { acciones. }\end{array}$ & $\begin{array}{l}\text { Capital de operación } \\
\text { - } \quad \text { Relación con los clientes } \\
\text { - Información de primera clase }\end{array}$ \\
\hline & $\begin{array}{l}\text { DIRECCIONADORES que proveen impulso para las } \\
\text { acciones. }\end{array}$ & $\begin{array}{ll}\text { - } & \text { Motivación del personal } \\
\text { - } \quad \text { Demandas de los clientes } \\
\text { - }\end{array}$ \\
\hline & $\begin{array}{l}\text { MECANISMOS que hacen posible realizar las } \\
\text { acciones. }\end{array}$ & $\begin{array}{ll}\text { - Inversión de capital } \\
\text { - } \quad \text { Infraestructura } \\
\text { - Organización del trabajo } \\
\text { - } \quad \text { Prácticas de la empresa } \\
\text { - Estructura de la empresa }\end{array}$ \\
\hline
\end{tabular}


Esta variable puede ser medida a través de las finanzas modernas (método de descuento de flujos de efectivo futuro, capitalización de ganancias, múltiplo de ingresos, valuación de activos netos, EVA, etc.) las mismas que deben ser consideradas para ajustar los estados financieros y reflejar la realidad financiera y económica para la toma de decisiones empresariales. Con independencia del modelo que se utilice, en todo proceso de valoración, se necesitan fuentes de información lo más objetivas posibles acerca de la empresa, a fin de comparar con un eventual precio de mercado el valor que ésta pueda tener para cada sujeto o usuario, ya sea en mercados organizados o en la negociación privada. Así pues, antes de aplicar cualquier método de valoración hará falta cuantificar una serie de circunstancias referentes a la empresa, unas más cuantitativas que otras y muchas de ellas, además, en forma prospectiva. Sin el trascendental proceso de cuantificación, cualquiera de las pretenciosas fórmulas de valoración de empresas o de acciones no deja de ser una entelequia (posición de su perfección: Aristóteles).
La información y sus consecuencias son a un mismo tiempo origen y destino de la valoración, input y output, es decir, elementos que posibilitan el proceso de valoración y que, a su vez, deben ser correctamente valorados. El primer aspecto conforma a la información como un factor productivo, que se sumaría a los clásicos capital y trabajo. Las normas contables proponen que sean contabilizados como gastos del ejercicio". El segundo aspecto, la información como recurso productivo susceptible de valoración, es objeto de los más novedosos estudios y muestra una complicación tal que aún no ha sido resuelta de forma satisfactoria la obtención "de alguna medida directa del valor de reposición de los intangibles...”. A su vez, el cálculo que resulte de dicha resolución es un dato que ha de utilizarse sucesivamente como input de un nuevo proceso valorativo.

Una vez definida la información como input (recurso) y output (activo), procede una clasificación más exhaustiva, dentro del primer aspecto, según el origen o fuente (interna o externa), según el nivel de registro (contable o extracontable) y según el fenómeno a que se refiera.

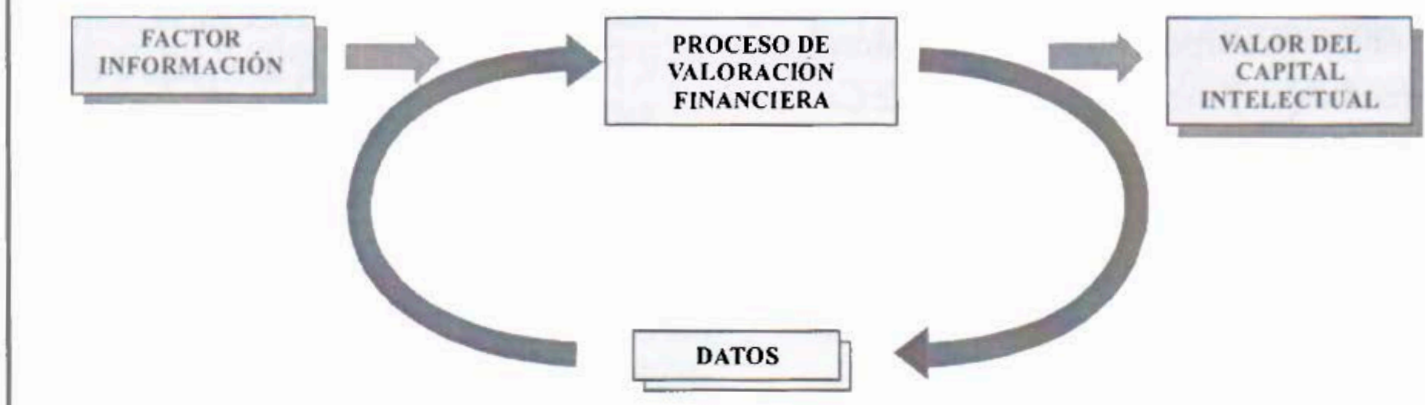

Figura 1. Woble importancia de la intormacion en la valoracion ae empresas. 
En conclusión, nuestra propuesta se sustenta en un nuevo enfoque contable a través del modelo sugerido para, posteriormente, medir en ella la performance o desempeño que tiene un efecto cuantitativo en la posición empresarial o valor de la empresa a una fecha determinada. De esta valorización empresarial sostenemos que se pueden determinar ajustes a los estados financieros tradicionales para llevarlos a valores económicos reales, además de detallarlos y explicarlos a través de una información complementaria que planteamos seguidamente.

Es decir, este modelo sugerido debe integrar al balance tradicional el valor del capital intelectual. Es decir, los contadores debemos de comenzar a analizar estas variables para buscar satisfacer a nuestros usuarios con la información que necesitan para la toma de decisiones empresariales.

b) Resumen del modelo sugerido

- Enfocar el capital intelectual a partir de la contabilidad financiera tradicional, de modo que lo que está registrado como gastos del período sea identificado como un elemento potencial del capital intelectual (ver modelo en anexo).

- Conocer la estrategia global de la empresa, sus objetivos corporativos y los indicadores de medición estimados.

- Medir la performance o desempeño a partir de la estrategia global de la empresa, sus objetivos corporativos, los indicadores de medición, y mediante la Matriz de Calificación.

La Matriz de Calificación es estimativa y depende del objetivo corporativo y la estrategia global del negocio, en ella se plantean los objetivos corporativos específicos, las estrategias específicas y los indicadores estimativos, con un peso cuantitativo que será direccionado por la Gerencia de acuerdo a la importancia para el cumplimiento del objetivo corporativo global previsto. Cuando éste se contraste con los resultados reales obtenidos, obtendremos un resultado que, de acuerdo a la matriz de calificación de resultados, determinará si los elementos del capital intelectual tienen un efecto positivo o negativo en el posicionamiento empresarial o la valorización de la empresa.

- Si determinamos que el posicionamiento empresarial es positivo, los gastos registrados en el período deben ser contabilizados como inversión para presentar el Balance del capital intelectual. Si es negativo lo aconsejable sería valorizar directamente a la empresa desde el punto de vista económico.

- Sr la empresa desea conocer su posicionamiento o valor de mercado, deberá de valorizarlo con la finalidad de ajustar las cifras de los componentes afectados para llegar a determinar valores reales del negocio.

c) Presentación del modelo de información complementaria del capital intelectual.

El modelo contable sugerido conlleva al reconocimiento o identificación que debe hacer el Contador Público de todos los gastos contabilizados que conllevan a maximizar la performance de la empresa $y$, por ende, crear valor agregado a través del capital intelectual.

Siendo éste el primer paso que debe considerar el Contador Público para identificar los elementos del capital intelectual que intervienen en la contabilidad tradicional, para cumplir con este primer paso, tomamos en cuenta el trabajo que fuera presentado en la 
Conferencia Interamericana de Uruguay, titulado "El Capital Intelectual- análisis crítico en el marco de las nuevas necesidades", que en el capítulo $\mathrm{V}$ presentó el formulario titulado: Capital intelectual-información complementaria a los estados contables. (Ver Anexo N. ${ }^{\circ} 01$ ).

En cumplimiento de uno de los objetivos del estudio de investigación, se distribuyó en el segundo semestre del año 2003, a 100 contadores públicos, el siguiente cuestionario de investigación 2003, cuyo contenido dice:

\section{CUESTIONARIO DE INVESTIGACIÓN 2003-CAPITAL INTELECTUAL-INFORMACIÓN COMPLEMENTARIA A LOS ESTADOS CONTABLES AL 31 DE DICIEMBRE DEL 2002}

1. El formulario: Capital intelectual-Información complementaria a los estados contables en el que sólo se han colocado dos columnas de Base Contable-2003 y Base Extracontable- 2003; la primera columna para mostrar información contable al cierre 2003 procedente de los libros de la empresa, en la medida que se puedan obtener; y lo más importante, la segunda columna para anotar la información extracontable, al cierre 2003, de aquellos rubros que consideren que la empresa los tiene, los tendrá o puede autogenerarlos basándose en el futuro de sus actividades o cambios que sean indispensables para lograr el desarrollo y la prosperidad de la empresa.

2. Su información es confidencial, pues no tiene que anotar el nombre de la empresa; sírvase devolverla al investigador que le ha pedido su colaboración antes del martes
02 de marzo de 2004, fecha en que el investigador tiene que presentar el informe final de sus encuestas.

El formulario adjunto al Cuestionario de Investigación 2003, que se muestra en el Anexo N.o 2, contiene solamente la "Base Contable Año 2003", y la "Base Extracontable Año 2003", sin buscar la comparabilidad con el año anterior 2002, con la finalidad de simplificar el proceso de las encuestas y facilitar un rápido retorno de los formularios con la información solicitada.

Como resultado de las encuestas, se recibieron 80 formularios con datos contables $y$ extracontables del año 2003. Se incluye en el presente informe del estudio de investigación un formulario de datos recibidos que se muestra en el Anexo N. ${ }^{\circ} 03$ con respectivas notas aclaratorias adjuntadas en el Anexo N. ${ }^{\circ} 04$.

El análisis de los formularios recibidos revela lo siguiente:

- La mayoría de los contadores públicos llenaron con buena información la columna Base Contable-Año 2003; pero la información de la columna Base Extracontable-Año 2003 es reducida.

- Esta información reducida, proporcionada por los contadores públicos, demuestra que no tienen un conocimiento cabal de los procesos del capital intelectual.

- Otra causa de esta información reducida de la columna Base ExtracontableAño 2003 podría ser producto de la formación universitaria sobre contabilidad tradicional, que se sustenta en los libros y documentos de la empresa, lo que los inhibe a proyectarse en dar información extracontable proveniente de otras fuentes. 
- Por último, una minoría de contadores públicos no colocó información alguna en la columna Base Extracontable-Año 2003 , lo que confirma un desconocimiento casi total de los procesos del capital intelectual.

\section{b) Modelo de matriz de calificación del capital intelectual}

Nuestro modelo exige que el contador público promueva su participación en el planeamiento estratégico que realiza la empresa, con la finalidad de tomar conocimiento de la visión, misión, objetivos corporativos, estrategias y planes que involucren a los elementos del capital intelectual.

Con este conocimiento, podrá generar la primera parte de la matriz que es estimativa y ponderada de acuerdo con los objetivos corporativos de la empresa. En la evaluación de la gestión del capital intelectual podrá involucrar su monitoreo a un cuadro de mando integral o Balanced Scorecard.

Al final del período económico, debemos de calificar su cumplimiento real como parte de la medición de la performance o desempeño, considerando para ello la comparación entre indicadores de medición estimativos y reales.

\section{CONCLUSIONES}

1. La bibliografía, separatas y artículos de revistas especializadas, provenientes de otros países con avanzada tecnología contable, confirman que los estudios sobre capital intelectual tienen un notable avance; mientras que en el Perú no se ha planificado una adecuada difusión a base de seminarios, fórums, conferencias, etc.; ni se han publicado libros con el tema del capital intelectual aplicado a realidades del entorno empresarial nacional.
2. Indudablemente, a nivel internacional se está avanzando en información adicional sobre el capital intelectual, dada la inquietud permanente en el ámbito de los contadores públicos de buscar los caminos que permiten cuantificar los datos obtenidos de la empresa, para presentarlos como información necesaria para la toma de decisiones sobre los componentes del capital intelectual. En el Perú no hay interés aún por estudiar tales componentes, ni para buscar la cuantificación como elemento necesario informativo.

3. Es un deber para los contadores públicos y estudiantes universitarios de todas las facultades del sistema de la universidad peruana, exigir el dictado de cursos, conferencias y seminarios sobre contabilidad del conocimiento para una adecuada administración del capital intelectual, tanto a los colegios profesionales como a sus propias universidades, para asimilar las innovaciones contables y extracontables que se están presentando. Así, los contadores públicos del Perú estarán capacitados en ofrecer nuevos servicios profesionales competitivos, aportando planteamientos de información contable de avanzada. De igual forma, los estudiantes universitarios, futuros contadores públicos, al salir de la universidad tendrán una adecuada preparación sobre este interesante tema profesional y estarán preparados para enfrentarse al reto de información futura que exigirán las empresas que hayan captado la importancia de recibir información contable sobre capital intelectual.

4. También tiene prioridad conocer los avances logrados sobre capital intelectual, realizando serios estudios de los trabajos nacionales que tratan este tema; $y$ de los artículos y libros que difunden los alcances 
logrados en las aplicaciones contables y extracontables a nivel empresarial.

5. El reto más ambicioso será buscar la mejor aplicación del capital intelectual en la economía peruana, para producir mayor información confiable en los estados financieros que preparen los contadores públicos, que permita el progreso y crecimiento financiero y económico de las empresas del Perú, el incremento de puestos de trabajo y la mejora de los niveles económicos de la población peruana. Nuestro enfoque presentado apunta hacia ese horizonte prometedor de mejora continua de la profesión contable.

\section{REFERENCIAS}

1. Bértora, Héctor Raúl, Llave de negocio, Ediciones Macchi López, 1975.

2. Chávez, Osvaldo; Pahlen Acuña, Ricardo. Valor llave. Un enfoque actual, Ediciones Macchi, 1996.

3. Boletín asociación española de contabilidad y auditoría N.o 50. Entrevista a Leandro Cañibano. "Nuevos desafíos de la contabilidad ante el siglo XXI".

4. Sierra Fernández, Monserrat. “ ¿Hacia una nueva contabilidad? Contabilidad del Capital Intelectual" Boletín de la Asociación Española de Contabilidad y Auditoría N.o 49.
5. International federation of accountants. Estudio 7: The measurement and management of Intellectual Capital: An introduction. EE.UU. Septiembre de 1998.

6. Cañibano, Leandro; García-Ayuso Covarsi, Manuel; Sánchez, Paloma. La relevancia de los intangibles para la valoración y la gestión de empresas: revisión de literatura . Asociación Española de Contabilidad y Auditoría. Madrid, 1999.

7. Carazay Cristina; Fernández, Analía; Nannini, María Susana; Suardi, Diana. La llave de negocios en los Estados Contables. Revista Desarrollo y Gestión N.o 10 Julio, 2000.

8. Bueno Campos, Eduardo (1998): "El capital intangible como clave estratégica en la competencia actual". Boletín De Estudios Económicos, vol. LIII, N.o 164 Agosto 1998, pp. 207-229.

9. Caballer Mellado, V. (1994): Métodos de valoración de empresas. Pirámide.

10. Cabrera Monroy, Francisca y Galindo Lucas, Alfonso (2000): "La importancia de la Información en la Valoración de Empresas". En la empresa del Siglo XXI: finanzas, tecnologías y sistemas de información (Volumen I), pp. 555564. Diputación de Cádiz. Jerez de la Frontera. 
ANEXO N. ${ }^{\circ} 1$

\begin{tabular}{|c|c|c|c|c|c|c|c|c|}
\hline \multicolumn{9}{|c|}{$\begin{array}{l}\text { CAPITAL INTELECTUAL } \\
\text { INFORMACIÓN COMPLEMENTARIA A LOS ESTADOS CONTABLES }\end{array}$} \\
\hline \multirow{2}{*}{$\begin{array}{l}\text { COMPONENTES DEL } \\
\text { CAPITAL INTELECTUAL }\end{array}$} & \multicolumn{8}{|c|}{ INDICADORES DEL EJERCICIO } \\
\hline & \multirow[b]{2}{*}{ DESCRIPCIÓN DEL INDICADOR } & \multirow{2}{*}{$\begin{array}{l}\text { Unidad } \\
\text { de } \\
\text { Medida }\end{array}$} & \multicolumn{2}{|c|}{$\begin{array}{c}\text { BASE } \\
\text { CONTABLE }\end{array}$} & \multicolumn{2}{|c|}{$\begin{array}{l}\text { BASE EXTRA } \\
\text { CONTABLE }\end{array}$} & \multicolumn{2}{|c|}{ BASE MIXTA } \\
\hline CLASIFICACIÓN & & & Año 01 & Año 00 & Año 01 & Año 00 & Año 01 & Año 00 \\
\hline \multicolumn{9}{|l|}{ 1. Mercado } \\
\hline \multicolumn{2}{|r|}{ Marcas de productos y servicios registrados } & Q & & & $\mathrm{X}$ & $\mathrm{x}$ & & \\
\hline & Inversión en adquisición de marcas & s & $\mathrm{x}$ & $\mathrm{x}$ & & & & \\
\hline Posición competitiva & Tamaño total del mercado primario en que opera la empresa & $\$$ & & & $\mathrm{x}$ & $\mathrm{x}$ & & \\
\hline & $\begin{array}{l}\text { Participación en el mercado primario en el que opera la empresa } \\
\text { Inversión en desarrollo de nuevos mercados }\end{array}$ & $\begin{array}{l}\% \\
5\end{array}$ & $\mathrm{x}$ & $\mathrm{x}$ & $\mathrm{x}$ & $\mathrm{x}$ & & \\
\hline $\begin{array}{l}\text { Administración } \\
\text { de clientes }\end{array}$ & Número tocal de clientes & $\dot{Q}$ & & & $\mathrm{x}$ & $\mathrm{X}$ & $\mathrm{x}$ & $\mathrm{x}$ \\
\hline & Clientes ganados & $\stackrel{0}{0}$ & & & $\mathrm{x}$ & $\mathrm{x}$ & $\boldsymbol{\lambda}$ & $\lambda$ \\
\hline & Clientes perdidos & $\mathrm{Q}$ & & & $\mathrm{x}$ & $\mathrm{x}$ & & \\
\hline & Inversión en servicios a clientes $\mathrm{N}^{\circ}$ total de clientes & $\mathrm{s}$ & & & & & & $\mathbf{x}$ \\
\hline & Indice de sarisfacción de clientes segrin encuesta (i) & $\%$ & & & $\mathrm{x}$ & $\mathrm{x}$ & & \\
\hline Nuevos productos & $\begin{array}{l}\text { Clemtes con mass de cinco años de ancigüedad } \\
\text { Ingresos provenientes de nuevos nemecios (menores a } 2 \text { años) }\end{array}$ & $\begin{array}{l}Q \\
\$\end{array}$ & $\mathrm{x}$ & $\mathrm{x}$ & & $\mathrm{x}$ & & \\
\hline y servicios (negrcios) & Ualidad neta proveniente de nuevos negocios & $\$$ & $\mathrm{x}$ & $\mathrm{X}$ & & & & \\
\hline & Nuevos productos y servicios lanzados & Q & & & $\mathrm{x}$ & $\mathrm{x}$ & & \\
\hline & Proporción ventas nuevos negocios $\mathrm{N}^{\circ}$ total de ventas & $\%$ & & & $\mathrm{x}$ & $\mathrm{x}$ & & \\
\hline Inversión en publicidad & Inversión total en publicidad & $\$$ & $\mathrm{x}$ & $\mathrm{x}$ & & & & \\
\hline & Inversión en publicidad de nucvos productos y servicios & s & $\mathrm{x}$ & $\mathrm{x}$ & & & & \\
\hline \multicolumn{9}{|l|}{2 Innovación y Tecnologia } \\
\hline Inversión en & Inversión total en investigación y desarrollo & $\$$ & $\mathrm{X}$ & $\mathrm{X}$ & & & & \\
\hline investigación y desarrollo & Recursos humanos dedicados a I \& $\mathrm{D}$ sobre recursos totales & $\%$ & & & $\mathrm{x}$ & $\mathrm{x}$ & & \\
\hline Obtención y de sarrollo & Cantidad de patentes y licencias obtenidas & $\mathrm{Q}$ & & & $\mathrm{x}$ & $\mathrm{x}$ & & \\
\hline de patentes y licencias & Inversión en la adquisición de patentes y licencias & $\$$ & $\mathrm{x}$ & $\mathrm{X}$ & & & & \\
\hline & Antigüedad promedio de patentes $y$ licencias & $\mathrm{T}$ & & & $\mathrm{x}$ & $\mathrm{x}$ & & \\
\hline & Patentes solicitadas pendientes de obtención & Q & & & $\mathrm{x}$ & $\mathrm{x}$ & & \\
\hline Capacidad de innovación & Tasa histórica de nuevos productos que llegar al mercado & $\%$ & & & $\mathrm{x}$ & $\mathrm{x}$ & & \\
\hline & Tiempo promedio de desarrollo $y / o$ idenofic. de un nuevo produc. & $\mathbf{T}$ & & & $\mathbf{x}$ & $\mathrm{x}$ & & \\
\hline & Proporción de empleados menores de 40 años & Q & & & $\mathrm{x}$ & $\mathrm{x}$ & & \\
\hline Sisternas de información & Inversiones en hardware & $\$$ & $\mathrm{x}$ & $\mathrm{x}$ & & & & \\
\hline & Inversiones en sof fware: Desarrollos propios & $\$$ & $\mathrm{x}$ & $\mathrm{x}$ & & & & \\
\hline & Inversiones en software: adquisición de & $\$$ & $\mathrm{x}$ & $\mathrm{x}$ & & & & \\
\hline & computadoras personales por empleado & $\mathrm{Q}$ & & & $\mathrm{x}$ & $\mathrm{x}$ & & \\
\hline \multicolumn{9}{|c|}{ 3. Competencias del personal } \\
\hline Desarrollo de talentos, & Inversión en formulación y desarrollo & & & & & & & \\
\hline destrezas y comperencias & de competencias par empleado & $\mathbf{s}$ & & & & & $\mathrm{x}$ & $\mathrm{x}$ \\
\hline & Horas de capacitación por empleado & $\mathrm{T}$ & & & $\mathrm{x}$ & $\mathrm{x}$ & & \\
\hline Eficiencia & Número de empleados & $Q$ & & & $\mathrm{x}$ & $\mathrm{X}$ & & \\
\hline & Ventas por empleado & $\$$ & & & & & $\mathrm{x}$ & $\mathrm{x}$ \\
\hline & Valor agregado por empleado & $\$$ & & & & & $\mathrm{x}$ & $\mathrm{x}$ \\
\hline & Beneficios totales $\mathrm{N}^{\circ}$ de empleados & $\$$ & & & & & $\mathrm{x}$ & $\mathrm{x}$ \\
\hline Retención del personal & Indice de satisfacción de empleados según encuesta (1) & $\%$ & & & $\mathrm{x}$ & $\mathrm{x}$ & & \\
\hline & Rotación de empleados & $\%$ & & & $\mathrm{X}$ & $\mathrm{X}$ & & \\
\hline Calificación del personal & Inversión en capacitación en nuevas tecnolog. y métodos de trab. & $\$$ & $\mathrm{x}$ & $\mathrm{x}$ & & & & \\
\hline & Porcentaje profesionales + técnicos $/ \mathrm{N}^{\circ}$ empleados & $\%$ & & & $\mathrm{x}$ & $\mathrm{x}$ & & \\
\hline & Nivel de educación promedio del personal & $Q$ & $\mathrm{x}$ & $\mathrm{x}$ & $\mathrm{X}$ & $\mathrm{x}$ & & \\
\hline
\end{tabular}

Referencia sobre la unidad de medida: T Tiempo, \% Porcentaje, Q Cantidad, \$Importe (i), Incorporar ficha récnica de las encuestas realizadas. 
ANEXO N. ${ }^{\circ} 2$

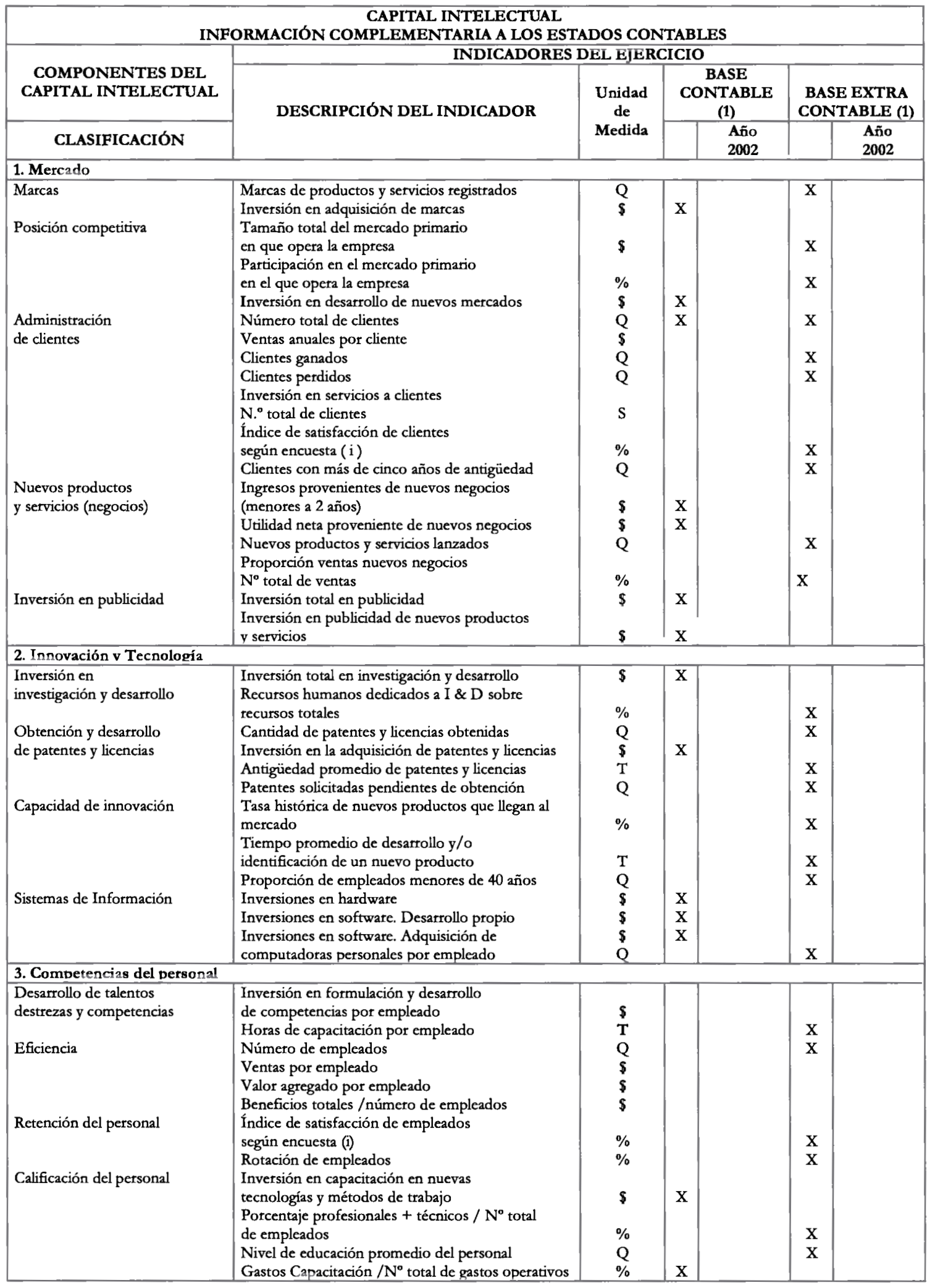

Referencia sobre la unidad de medida: T Tiempo, \% Porcentaje, Q Cantidad, \$ Importe (i), Incorporar ficha técnica de las encuestas realizadas. (1) Las matcas " $\mathrm{X}$ " sirven para indicar los rubros que solicitan información 
ANEXO N. ${ }^{\circ} 3$

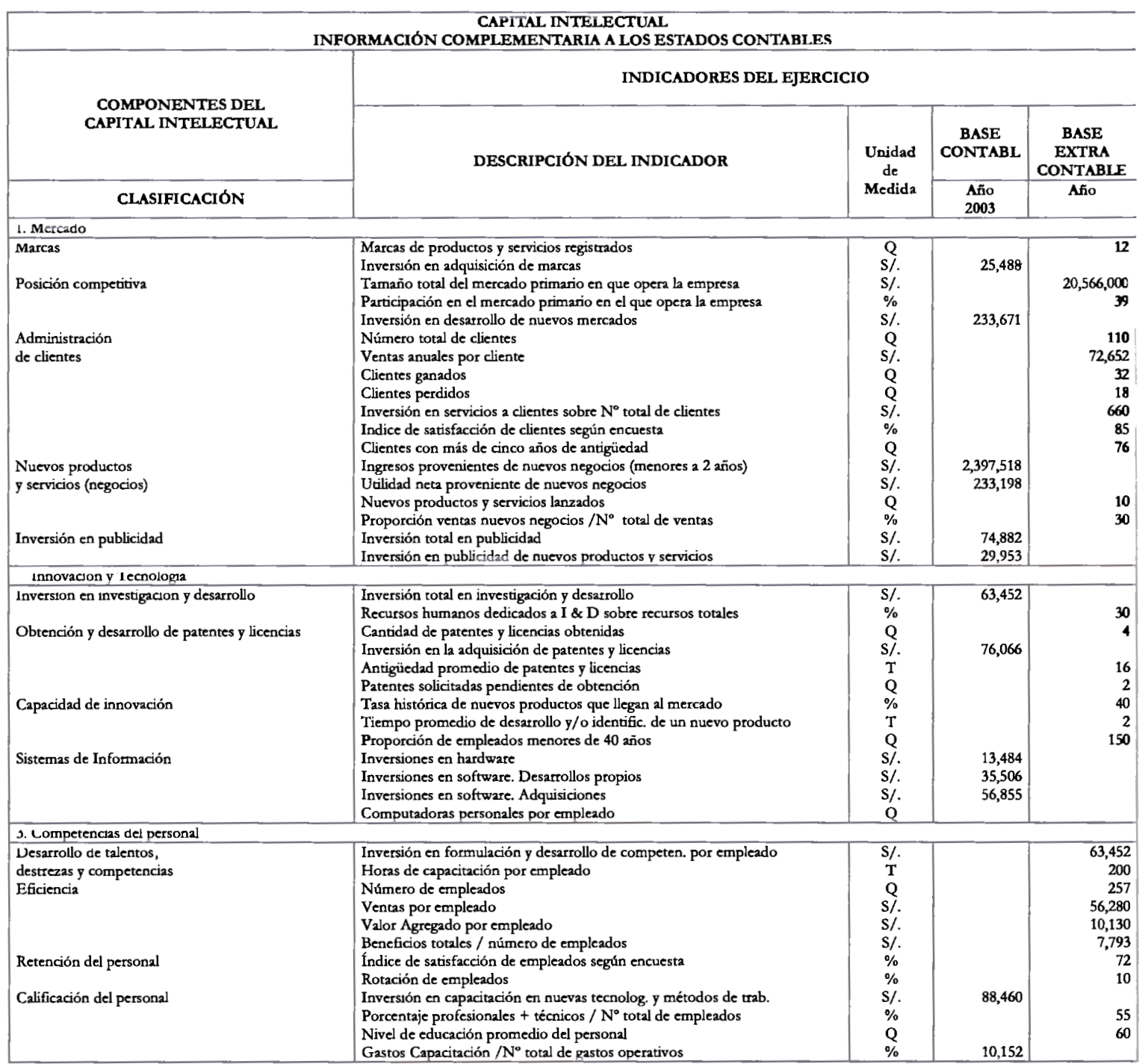




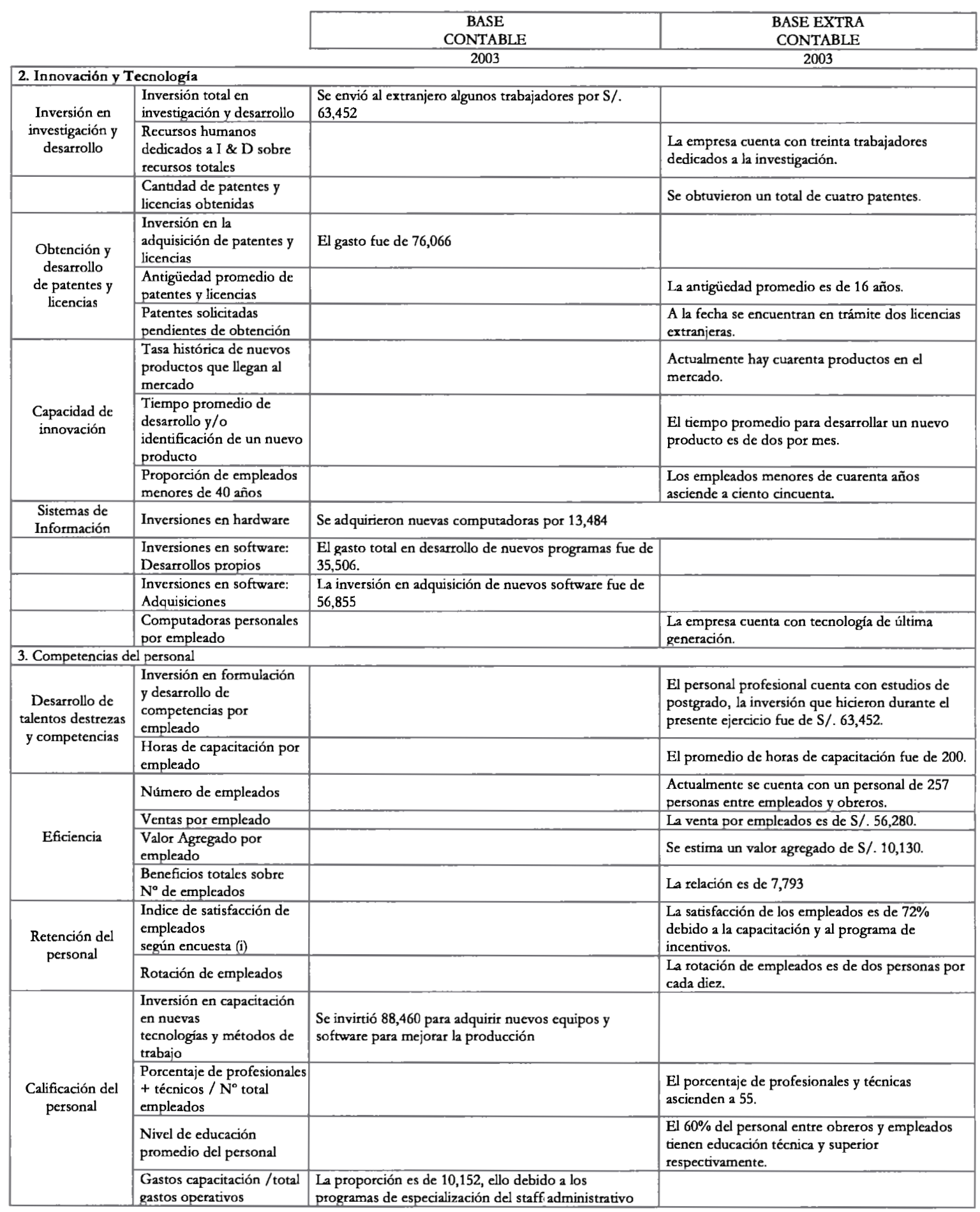


Empresa

: "La textil SA"

ANEXO No 4

Objetivo Corporativo: "Incrementar la rentabilidad del período en un $5 \%$

Estrategia Global : Incrementar la participación en el mercado, considerando la incursión de nuevos productos en el mercado, incremento de las ventas por cliente, etc.

\section{MATRIZ DE CALIFICACIÓN DEL CAPITAL INTELECTUAL}

Al 31 de diciembre de 2003

\begin{tabular}{|c|c|c|c|c|}
\hline $\begin{array}{l}\text { COMPONENTES DEL CAPITAL } \\
\text { INTELECTUAL }\end{array}$ & $\begin{array}{c}\text { OBJETIVOS } \\
\text { CORPORATIVOS }\end{array}$ & $\begin{array}{l}\text { MEDICION DE } \\
\text { INDICADORES }\end{array}$ & $\begin{array}{l}\text { PESO } \\
\text { CUANTITATIVO } \\
\text { ESTIMADOS }\end{array}$ & $\begin{array}{l}\text { CALIFICACIÓN } \\
\text { CUANTITATIVA } \\
\text { REAL }\end{array}$ \\
\hline \multicolumn{5}{|l|}{ 1. Mercado } \\
\hline - Administración de clientes & $\begin{array}{l}\text { - Incremento de las ventas } \\
\text { en un } 5 \% \\
\text { - Incremento de las ventas } \\
\text { por diente } \\
\text { - Aumento de clientes en un } \\
5 \% \\
\text { - Disminución en la } \\
\text { proporción de mercaderías } \\
\text { devueltas }\end{array}$ & $\begin{array}{l}\text { - Ventas Netas/Nentas } \\
\text { totales del sector } \\
\text { - Ventas Netas/Total de } \\
\text { clientes } \\
\text { - Clientes nuevos/Total de } \\
\text { clientes } \\
\text { - Valor de la mercadería } \\
\text { devuelta/Nentas Netas }\end{array}$ & 10.00 & 13.50 \\
\hline - Posición competitiva & $\begin{array}{l}\text { Invertir 3\% de la utilidad } \\
\text { estimada en el desarrollo } \\
\text { de nuevos mercados }\end{array}$ & & & \\
\hline - Nuevos productos y servicios & $\begin{array}{l}\text { - Lanzar cínco nuevos } \\
\text { productos y servicios }\end{array}$ & & & \\
\hline - Inversión en publicidad & $\begin{array}{l}\text { - Se debe publicitar los } \\
\text { nuevos productos en } \\
\text { canales de TV y emisoras } \\
\text { que llegan al sector } \\
\text { económico A }\end{array}$ & & & \\
\hline \multicolumn{3}{|l|}{ I. PUNTAJE PARCIAL } & 50.00 & 45.00 \\
\hline \multicolumn{5}{|l|}{ 2. Innovación y tecnología } \\
\hline \multirow[t]{3}{*}{$\begin{array}{l}\text { Inversión en investigación, } \\
\text { desarrollo de nuevos productos, } \\
\text { patentes y licencias }\end{array}$} & $\begin{array}{l}\text { Los gastos de } \\
\text { investigaciốn y desarrollo } \\
\text { deben ser efectivos }\end{array}$ & $\begin{array}{l}\text { - Utilidad Neta acumulada } \\
\text { para nuevos productos/ } \\
\text { gastos de inversión y } \\
\text { desarrollo acumulado }\end{array}$ & 10.00 & 8.00 \\
\hline & $\begin{array}{l}\text { - Los nuevos productos que } \\
\text { se comercializan deben } \\
\text { incrementar nuestros } \\
\text { ingresos }\end{array}$ & $\begin{array}{l}\text { - Ingresos acumulados } \\
\text { por nuevos productos/ } \\
\text { total de ingresos } \\
\text { anuales }\end{array}$ & 7.50 & 7.00 \\
\hline & $\begin{array}{l}\text { - La supervivencia de los } \\
\text { productos nuevos en los } \\
\text { últimos tres años }\end{array}$ & $\begin{array}{l}\text { - Cantidad de productos } \\
\text { nuevos/Cantidad total } \\
\text { de productos }\end{array}$ & 7.50 & 7.00 \\
\hline $\begin{array}{l}\text { - Inversión en sistemas de } \\
\text { información }\end{array}$ & & & & \\
\hline - Capacidad de innovación & & & & \\
\hline \multicolumn{3}{|l|}{ II. PUNTAJE PARCIAL } & 25.00 & 22.00 \\
\hline \multicolumn{5}{|l|}{ 3. Competencias del personal } \\
\hline $\begin{array}{l}\text { - Retención del personal y } \\
\text { calificación }\end{array}$ & $\begin{array}{l}\text { Promover el incremento de } \\
\text { ventas por empleado en } \\
\text { un } 5 \% \\
\text { Los costos de la promover } \\
\text { las ventas deben disminuir } \\
\text { en un } 2 \% \text { en relación al } \\
\text { período anterior. } \\
\text { La capacitación de } \\
\text { empleados debe alcanzar } \\
\text { al total de ellos. } \\
\text { Las quejas por el ambiente } \\
\text { laboral deben disminuir en } \\
\text { un } 5 \%\end{array}$ & $\begin{array}{l}\text { - Ventas Netas/No de } \\
\text { empleados } \\
\text { - Utilidad Neta/No de } \\
\text { empleados } \\
\text { - No de empleados } \\
\text { capacitados/total } \\
\text { empleados } \\
\text { - No de quejas/cantidad } \\
\text { de empleados total }\end{array}$ & 10.00 & 8.00 \\
\hline $\begin{array}{l}\text { - Desarrollo de talentos, } \\
\text { destrezas y competencias }\end{array}$ & $\begin{array}{l}\text { Inversión en la } \\
\text { formulación y desarrollo } \\
\text { de competencias }\end{array}$ & & & \\
\hline \multicolumn{3}{|l|}{ III. PUNTAJE PARCIAL } & 25.00 & 22.00 \\
\hline & & PUNTAJE TOTAL & 100.00 & $* 89.00$ \\
\hline
\end{tabular}

